\title{
Neuroprotective effects of crocin I and II in an ischemia-reperfusion injury model
}

Baowei Lv ${ }^{1}$, Junyan Yin ${ }^{1}$, Chunqing Feng ${ }^{2, *}$, Yanhui $\mathrm{Li}^{2}$

1 Department of Traditional Chinese Medicine, Liaocheng People's Hospital, Liaocheng 252000, China

2 Department of Rehabilitation, Liaocheng People's Hospital, Liaocheng 252000, China

*Corresponding Author: Dr. Chunqing Feng

Department of Rehabilitation, Liaocheng People's Hospital, Liaocheng 252000, China

\section{Address:}

\#67 Dongchang West Road, Liaocheng 252000, China;

E-mail: $13508939361 @ 163 . c o m$.

\section{Running title: crocin I and II in hypoxia}

\begin{abstract}
Background: Crocin I and II are derived from the medicinal plant Crocus sativus L. (Saffron), and their neuroprotective effects have been attracting more and more attention. However, their protective effect against cerebral apoplexy induced by hypoxia has not been reported. In this study, we aimed to clarify the roles of crocin I and II in protecting against ischemic injury.

Materials/Methods: We generated a rat cerebral ischemia-reperfusion injury model using a reversible cerebral artery occlusion suture method and found changes in amino acid neurotransmitters in the frontal cortex after drug administration. We also identified changes in mRNA expression of Bcl2, Bax, Casp3, P38, and NFkb1 in the frontal cortex and changes in antioxidant indices in the brain.

Results: Crocin I and II both had protective effects on ischemic/anoxic injury in vivo by downregulating the expression of Casp3 and $N f k b 1$ mRNA and the steady-state levels of excitatory amino acids/inhibitory amino acids during ischemia and reperfusion and by improving the total antioxidant capacity and total superoxide
\end{abstract}


dismutase activities during ischemia. We also found that crocin I and II had synergistic effects when used together.

Conclusions: These findings displayed that crocin I and II could protect animal model against ischemic and anoxic injury and provided new evidence for both molecules' potential medicinal value.

MeSH Keywords: crocin I; crocin II; middle cerebral artery occlusion model; cerebral ischemia-reperfusion injury 


\section{Background}

Ischemic stroke is a primary cause of mortality and morbidity worldwide [1] and is now the leading cause of death in China [2]. However, there are limited options for the treatment of ischemic stroke $[3,4]$, and only $2-5 \%$ of stroke patients receive thrombolytic therapy to restore blood flow because of the narrow post-stroke time window $(<4.5 \mathrm{~h})$ in which ischemic tissue can be rescued [5,6]. Recent advances in methods of treating ischemia-reperfusion, including neuroendovascular interventions, mechanical thrombectomy, in vivo imaging, stem cell therapy and computing programs, were developed.[7-10]. Animal studies have been performed to determine the pathological changes that occur after stroke and to explore the efficacy of new drugs and therapies.

The middle cerebral artery occlusion (MCAO) model in rats mimics the reperfusion of human ischemia after stroke and is used to study the pathological changes that occur after ischemic stroke and the mechanisms of drug action [11-13]. MCAO stroke induces cell death through interactions between excitatory amino acid (EAA) toxicity, acidosis, inflammatory responses, oxidative stress, peri-infarct depolarization, and apoptosis [14-16]. Both anoxia and ischemia increase the production of reactive oxygen species (ROS) [17-19] and induce inflammatory responses [19-21], and these cause damage to cellular components and negatively affect cell growth and function $[22]$.

Crocin I and II (Figure 1) are purified from the medicinal plant Crocus sativus L. (Saffron) [23] and are widely reported to have antineoplastic properties [24-26]. Crocins were also found have the effect on metabolic syndrome-induced osteoporosis in rats [27]. Their neuroprotective effects have been attracting increasing attention; for example, the potential therapeutic effect of crocins for Alzheimer's disease has been reported [23]. It has been also found that crocin could enhance hypothermia therapy in hypoxic ischemia-induced brain injury in mice [28]. The present study explored the 
efficacy and mechanisms of crocin I and II in the treatment of cerebral apoplexy induced by hypoxia in an MCAO model and investigated the neuroprotective effects of crocin I, crocin II, and a mixture of the two in in vivo models.

\section{Materials and methods}

\section{Animals}

Pathogen-free male SD rats (weight upon receipt: 120-140 g; weight at time of the experiments: 140-160 g) were provided by Beijing Vital River Laboratory Animal Technology Co., Ltd. (Beijing, China). The breeding environment consisted of a barrier-class animal laboratory [license number: SYXK(Lu)2013-0012] maintained at 20-25 ${ }^{\circ} \mathrm{C}$ with a $12: 12 \mathrm{~h}$ light/dark cycle. The laboratory animals were cared for according to "The Care and Use of Laboratory Animals" by Liaocheng People's Hospital.

\section{Rat cerebral ischemia-reperfusion injury model}

The experimental groups included the sham group (Sham), MCAO-only group (Model), crocin I group (C1), crocin II group (C2), and crocin I and II (1:1) mixture group $(\mathrm{C} 1+2)$. Both the Sham and Model groups were intragastrically administered $0.5 \%$ sodium carboxymethyl cellulose (Alibaba Co., Ltd., Hangzhou, China). The intragastrically administrated doses of crocin I (lot number: 111588-201303, purity: 92.6\%), crocin II (lot number: 111589-201103, purity: 91.9\%), and the crocin I and II mixture were $0.013 \mathrm{mg} \cdot \mathrm{kg}^{-1}$ per day, $0.015 \mathrm{mg} \cdot \mathrm{kg}^{-1}$ per day, and $0.014 \mathrm{mg} \cdot \mathrm{kg}^{-1}$ per day, respectively, which were similar to the clinically relevant dose in humans. The intragastric administration volume was $10 \mathrm{~mL} \cdot \mathrm{kg}^{-1}$ and was given once per day for 14 days. After the 14-day treatments, rats from the Model, C1, C2, and C1+2 groups were anesthetized using $10 \%$ chloral hydrate at a dosage of $3.5 \mathrm{~mL} \cdot \mathrm{kg}^{-1}$. The MCAO operation was conducted using a reversible cerebral artery occlusion suture method that was designed for rats [29]. The Sham group underwent surgery similar to that of the MCAO group, but the external carotid artery and branches were not actually blocked. 


\section{Amino acid sample preparation in the rat cortex}

Six rats were allocated to each of the Sham, Model, C1, C2, and C1+2 groups as described above. At 40, 70, and $100 \mathrm{~min}$ after the MCAO operation and at 40, 100, and $200 \mathrm{~min}$ after reperfusion (referred to as I-40, I-70, I-100, I-100+R-40, I-100+R-100, and I-100+R-200, respectively), one rat in each group was decapitated and their brains were removed by quickly stripping the cerebral tissues, cutting off approximately $25 \mathrm{mg}$ of frontal lobe tissue, and adding $1 \mathrm{~mL}$ of a methanol: water solution (volume ratio 1:1). The tissues were added to an ice bath with ultrasonication for $15 \mathrm{~min}$ and then homogenized by centrifugation at $13,000 \times g$ for $30 \mathrm{~s}$ at $4^{\circ} \mathrm{C}$. A portion of the supernatant $(0.5 \mathrm{~mL})$ was removed, and the solution containing the marker L-homoserine was added to reach a volume of $100 \mu \mathrm{g} / \mathrm{L}$. After $15 \mathrm{~min}$ of centrifugation at $13,000 \times g$ at $4^{\circ} \mathrm{C}$, the supernatant was extracted for immediate use in the derivatization reaction.

\section{High-performance liquid chromatography}

Glutamate (Glu) (lot number: 140690-201203, purity: 100\%) were purchased from the National Institutes for Food and Drug Control (Beijing, China). Taurine (Tau) (lot number: T103829, purity: 99\%), $\gamma$-aminobutyric acid (GABA) (lot number: A104200, purity: 99\%), ethanethiol (lot number: E110411, purity: 98\%), and o-phthalaldehyde (lot number: P108632, purity: 98\%) were purchased from Shanghai Aladdin Biochemical Technology Co. Ltd. (Shanghai, China). High-performance liquid chromatography was performed on an Agilent 1260 (Agilent Technologies Singapore [International] Pte. Ltd., Singapore, Singapore) with precolumn derivatization. The software version used was ChemStation (Revision) B.04.03 (16). The derivatization reagent consisted of $27 \mathrm{mg}$ of o-phthalaldehyde and $40 \mu \mathrm{L}$ of ethanethiol dissolved in $5 \mathrm{~mL}$ of methanol to which $5 \mathrm{~mL}$ of $0.1 \mathrm{mmol} / \mathrm{L}$ sodium tetraborate buffer solution was added. Normal concentrations of amino acid solutions or supernatants from the previous section ( $40 \mu \mathrm{L}$ ) were added to $20 \mu \mathrm{L}$ of the prepared derivatization solution. The solution was mixed for $30 \mathrm{~s}$, allowed to stand for $120 \mathrm{~s}$, and run through an 
Agilent ZORBAX C18 column $(4.6 \times 150 \mathrm{~mm}, 5 \mu \mathrm{m})$. Mobile phase A consisted of $10 \mathrm{mmol} / \mathrm{L} \mathrm{Na}_{2} \mathrm{HPO}_{4}: 12 \mathrm{H}_{2} \mathrm{O}$ (pH 6.88), and mobile phase $\mathrm{B}$ consisted of methanol/acetonitrile (3:1). The ratio of phases A and B in solution was 6.4:3.6. The excitation wavelength was $355 \mathrm{~nm}$, and the emission wavelength was $450 \mathrm{~nm}$. The gain margin was $\times 6$ with a lower sensitivity, the flow rate was set to $1.0 \mathrm{~mL} / \mathrm{min}$, the column temperature was $35^{\circ} \mathrm{C}$, and the sample size was $10 \mu \mathrm{L}$.

\section{Antioxidant index determination}

Ten rats each were allocated to the Sham, Model, C1, C2, and C1+2 groups as described above. In this experiment, the suture was taken out after $100 \mathrm{~min}$ of ischemia, and after reperfusion was performed for $20 \mathrm{~h}$ the rats were decapitated. The cerebral tissues were kept in cold normal saline to remove the blood and then were dried with filter paper. The left and right cerebral hemispheres were removed and weighed to prepare a $10 \%$ tissue homogenate. The kits used for detecting malondialdehyde (MDA) and glutathione peroxidase (GSH-Px) were purchased from Beijing Solarbio Science \& Technology Co., Ltd. (Beijing, China) and Sigma-Aldrich Corporation (St. Louis, MO, USA) respectively. The kits used for detecting total antioxidant capacity (T-AOC) and superoxide dismutase (SOD) were both from Nanjing Jiancheng Bioengineering Institute (Nanjing, China). The relevant index detection was conducted according to the instructions of the kits described above. A UV-2550 ultraviolet spectrophotometer (Shimadzu Scientific Instruments, Kyoto, Japan) was used for detection.

\section{Real-time polymerase chain reaction}

Twelve rats each were allocated to the Sham, Model, C1, C2, and C1+2 groups as described above. After the MCAO operation, one rat from each group was sacrificed by decapitation at I-40, I-100, I-100+R-40, and I-100+R-200. RNA Lyzol (Shanghai ExCell Biology, Inc., Shanghai, China) was used for RNA extraction. A RevertAid First Strand cDNA Synthesis Kit (Thermo Fisher Scientific Inc., Waltham, MA, USA) was used for reverse transcription, and $5 \mu \mathrm{g}$ of RNA was used for each sample. 
RT-PCR Master Mix (Toyobo Co., Ltd., Osaka, Japan) was used on an ABI 7500 fast real-time fluorogenic quantitative RT-PCR system (Applied Biosystems, Foster City, CA, USA). The primers used in the real-time polymerase chain reaction were P-P38-F: 5'-GA ATG GAA GAG CCT-3'; P-P38-R: 5'-GAC AGA ACA GAA GC-3'; NF-кB-F: 5'-CGA CAC CTC TAC AC-3'; NF-кB-R: 5'-GGC TCA AAG TTC TC-3'; BCL2-F: 5'-A CAA CAT CGC TCT-3'; BCL2-R: 5'-CA GGA GAA ATC AAA-3'; BAX-F: 5'-CCA CCA AGA AGC-3'; BAX-R: 5'-A CGG AAG AAG ACC-3'; caspase-3-F: 5'- CTT GGA AAG CAT C-3'; caspase-3-R: 5'-AGC CTG GAG CAC AG-3'; actin-F: 5'-TC TAT GAG GGT TAC-3'; and actin-R: 5'-GTC ACG CAC GAT TTC-3'.

\section{Statistical analysis}

All data are presented as the mean \pm standard deviation and were analyzed with GraphPad Prism 6.01 statistical software (GraphPad Software, Inc., La Jolla, CA, US). The t-test was used for analyzing measurement data. Differences between two groups were analyzed by using the Student's t-test. Comparison between multiple groups was done using One-way ANOVA test followed by Post Hoc Test (Least Significant Difference). $P<0.05$ was considered statistically significant.

\section{Results}

\section{Effects of crocin I and II on cortical amino acids in the rats with MCAO-induced} cerebral ischemia-reperfusion injury

Compared with the Sham group, the Glu concentrations in the Model group were significantly increased at each time point of cerebral ischemia and reperfusion (Figure 2A, $P<0.05$ ). Compared with the Model group, the Glu concentrations in the $\mathrm{C} 1, \mathrm{C} 2$, and $\mathrm{C} 1+2$ groups were decreased at the I-100 and I-100+R-40 time points, and in the $\mathrm{C} 2$ and $\mathrm{C} 1+2$ groups the Glu concentrations were decreased at the I-100+R-70 and I-100+R-200 time points (Figure 2A, $P<0.05$ ). We also found that Glu levels in the $\mathrm{C} 1+2$ group were lower than either the $\mathrm{C} 1$ or $\mathrm{C} 2$ groups at the $\mathrm{I}-100+\mathrm{R}-40$, I-100+R-70, and I-100+R-200 time points (Figure $\mathbf{2 A}, P<0.05$ ), suggesting that administration of crocin I and II at the same time has a stronger effect 
than using only one alone.

Compared with the Sham group, the Tau concentrations in the Model group were significantly increased at I-70 and I-100 (Figure 2B, $P<0.05$ ). Compared with the Model group, the Tau concentrations were decreased at all six time points in the $\mathrm{C} 1$, $\mathrm{C} 2$, and $\mathrm{C} 1+2$ groups (Figure $\mathbf{2 B}, P<0.05$ ). Notably, the Tau level in the $\mathrm{C} 1+2$ group was lower than either the $\mathrm{C} 1$ or $\mathrm{C} 2$ groups at I-100+R-40, I-100+R-70, and I-100+R-200 (Figure 2B, $P<0.05$ ).

Compared with the Sham group, the GABA concentrations in the Model group were significantly increased at I-70, I-100, I-100+R-40, I-100+R-70, and I-100+R-200 (Figure 2C, $P<0.05$ ). Compared with the Model group, the GABA concentrations in at least one treatment group were decreased at I-40, I-100, I-100+R-40, I-100+R-70, and I-100+R-200 (Figure 2C, $P<0.05$ ). Notably, the GABA level in the $\mathrm{C} 1+2$ group was lower than the $\mathrm{C} 1$ group at $\mathrm{I}-100+\mathrm{R}-70$ (Figure 2C, $P<0.05)$.

Effects of crocin I and II on antioxidant indices in MCAO-induced cerebral ischemia-reperfusion injury

None of the four antioxidant indices were significantly changed in the left-brain tissue (the non-ischemic side) of the rats in the Model group or crocin-administered groups compared with the Sham group (Figure 3,P>0.05). MDA levels were significantly increased in the right-brain tissue (the ischemic side) in the Model group (Figure 3A, $P<0.05$ ), while GSH-Px, T-AOC, and total SOD (T-SOD) levels were significantly decreased (Figure 3B-3D, $P<0.05$ ). Compared with the Model group, the T-AOC and T-SOD activities were significantly increased in the right-brain tissue of the $\mathrm{C} 1+2$ group (Figure 3B-3C, $P<0.05$ ).

Effects of crocin I and II on p38, Nfkb1, Bcl2, Bax, and Casp3 mRNA expression in the frontal lobe of rats with MCAO-induced cerebral ischemia-reperfusion injury 
The Bcl2/Bax ratio of mRNA expression was significantly decreased in the Model group compared with the Sham group at all four time points (Figure 4A, $\mathrm{P}<0.05$ ). Compared with the Model group, the ratio was not upregulated in the $\mathrm{C} 1, \mathrm{C} 2$, or $\mathrm{C} 1+2$ groups (Figure 4A, P>0.05). The Casp3 and Nfkb1 mRNA expression levels were significantly higher in the Model group compared to the Sham group (Figure 4B-4C, $P<0.05$ ), and they were downregulated in the $\mathrm{C} 1, \mathrm{C} 2$, and $\mathrm{C} 1+2$ groups at all four time points (Figure 4B-4C, $P<0.05$ ). At I-100+R-200, the Casp3 mRNA level in the $\mathrm{C} 1+2$ group was significantly lower than in the $\mathrm{C} 1$ group, while there were no differences between the $\mathrm{C} 1$ and $\mathrm{C} 2$ groups (Figure 4C, $P<0.05$ ). The $N f k b 1$ mRNA level in the $\mathrm{C} 1+2$ group was lower than in the $\mathrm{C} 1$ and $\mathrm{C} 2$ groups (Figure $4 \mathrm{C}, P<0.05$ ), and the p38 mRNA expression levels were unchanged among groups (Figure 4D, $P>0.05)$.

\section{Discussion}

We generated a rat cerebral ischemia-reperfusion injury model using a reversible cerebral artery occlusion suture method. Since crocins were used as preventative treatment for ischemia-reperfusion injury, they were actually administrated before ischemia-reperfusion injury modelling. We then further discussed the pathways involving inflammatory factors (p38 and NF-кB), apoptotic factors (Bcl-2, Bax, and caspase-3), amino acid neurotransmitters, and ROS that contribute to learning and memory in the cortex with drug intervention in order to elucidate the effects and properties of crocin I and II and thereby provided support for their clinical applications.

The neurotoxic properties of EAAs result in the initiation and development of cerebral tissue injury, and Glu is one of the most important EAAs in the brain [30]. Tau and GABA are important inhibitory amino acids (IAAs) in the brain, and in models of cerebral ischemia they are released by cells to counteract the effects of EAAs [31,32]. The present study was conducted to assess the neuroprotective mechanisms of crocin I and II by measuring changes in amino acid concentrations in the cerebral cortex at 
different time points after ischemia-reperfusion injury. The Glu/GABA ratio is a measure of the balance of cerebral EAAs and IAAs, and we found that in ischemia-reperfusion injury the in vivo Glu and GABA levels were increased (Figure $\mathbf{2 A}$ and $\mathbf{2 C}$ ) as were the corresponding overall EAA/IAA ratios. In all three crocin-treatment groups, the concentrations of certain amino acids that are involved in EAA/IAA homeostasis were downregulated at different time points and they had a tendency to approach normal levels. Thus crocin I and II appear to play a protective role in the acute stage of ischemia-reperfusion by reducing the neurotoxicity of EAAs. Crocin I and II decreased the concentrations of the IAAs Tau and GABA (Figure 2B-2C), and the Glu/GABA ratio was maintained at normal homeostatic levels and thus reduced the toxicity of EAAs. Maintaining and improving the relative concentrations of EAA and IAA might be one of the mechanisms underlying crocin I and II's action in reducing the cerebral infarction volume in acute cerebral ischemia.

The T-AOC of an organism reflects its ability to resist oxidation and to scavenge free radicals [33] and consists of enzymatic and nonenzymatic antioxidant defense systems that include, but are not limited to, SOD, catalase, GSH-Px, vitamin C, vitamin E, glutathione, glucose, and $\beta$-carotene. The T-AOC is more dependable for evaluating whether stress has caused oxidative damage to an organism than information provided by a single antioxidant index. When endogenous or exogenous events cause abnormalities in an organism's metabolism that lead to the sudden production of large amounts of ROS, the antioxidant defense system will be triggered and excessive ROS will be removed, thereby protecting the tissues from oxidative damage [34]. SOD is an endogenous antioxidant enzyme that metabolizes ROS into hypotoxic substances, hence protecting cells from damage and playing a crucial role in balancing oxidation and antioxidation in an organism [35,36]. Nonenzymatic ROS attacks on polyunsaturated fatty acids in biological membranes form lipid peroxides such as MDA, and thus the MDA concentration reflects the degree of lipid peroxidation and indirectly reflects the degree of cell damage. The current study also examined the antioxidation mechanisms of crocin I and II in protecting against ischemic cerebral 
injury and their influence on antioxidant indices in bilateral ischemic and non-ischemic rat cerebral tissues. Crocin I and II significantly improved T-SOD and T-AOC activity in bilateral ischemic cerebral tissues (Figure 3C-3D), suggesting that in vivo antioxidant enzyme synthesis rescues the aberrant oxidation/antioxidation balance seen in oxidative-stress injury and that crocin I and II play important roles in the enzymatic and nonenzymatic antioxidant defense systems to protect cells from oxidation damage.

We also found that when crocin I and II were administered prior to MCAO-induced ischemia-reperfusion injury in rats, the expression of Casp3 and Nfkbl mRNA was downregulated at different time points after ischemia reperfusion (Figure 4B-4C). These results suggest that crocin I and II play a protective role in ischemia-reperfusion injury through the downregulation of factors associated with apoptosis.

Interestingly, we also found that using crocin I and II together with both at half dosages had better effects at some time points than administering only crocin I or crocin II alone at the full dosage (Figure 2-4), thus suggesting that crocin I and II have synergistic neuroprotective effects against in vivo ischemic and anoxic injury.

To be honest, we also compared infract volumes between $\mathrm{C} 1+2$ and Model groups using TTC staining and in vivo MR imaging. Results exhibited no statistical significance (not shown) between groups for both methods. As crocins were designed as preventative treatment for ischemia-reperfusion injury in current study, they were administrated prior to modeling. Though crocins could not significantly reduce the infract volume caused by ischemia-reperfusion injury, they restored levels of important molecular makers of ischemia-reperfusion injury including amino acid neurotransmitters, antioxidant indexes, inflammatory factors and key molecules in apoptosis signaling. 


\section{Conclusions}

We found that crocin I and II exerted protective effects against in vivo ischemic and anoxic injury and exhibited synergistic effects when used together. This research contributes to a greater understanding of the mechanistic functions of crocin I and II. We will further evaluate the neural functioning, including behaviors such as modified neurological severity score (mNSS) and foot-fault degree, permeability of the blood brain barrier $\mathrm{BBB}$, in vivo morphology of brain and neurobiological marker staining for tissues of the infarction and surrounding area, to determine whether crocin protection improves prognosis in the future work.

\section{Conflict of interest None.}

\section{References}

1. Feigin VL, Lawes CM, Bennett DA, Anderson CS: Stroke epidemiology: a review of population-based studies of incidence, prevalence, and case-fatality in the late 20th century. Lancet Neurol, 2003; 2: $43-53$

2. Zhang LF, Yang J, Hong Z et al: Proportion of different subtypes of stroke in China. Stroke, 2003; 34: 2091-6

3. Mozaffarian D, Benjamin EJ, Go AS et al: Heart Disease and Stroke Statistics-2016 Update: A Report From the American Heart Association. Circulation, 2016; 133: e38-360

4. Truelsen T, Heuschmann PU, Bonita R et al: Standard method for developing stroke registers in low-income and middle-income countries: experiences from a feasibility study of a stepwise approach to stroke surveillance (STEPS Stroke). Lancet Neurol, 2007; 6: 134-9

5. Hacke W, Kaste M, Bluhmki E et al: Thrombolysis with alteplase 3 to 4.5 hours after acute ischemic stroke. N Engl J Med, 2008; 359: 1317-29

6. Del ZG, Saver JL, Jauch EC, Adams HJ: Expansion of the time window for treatment of acute ischemic stroke with intravenous tissue plasminogen activator: a science advisory from the American Heart Association/American Stroke 
Association. Stroke, 2009; 40: 2945-8

7. Chimowitz MI: Endovascular treatment for acute ischemic stroke--still unproven. N Engl J Med, 2013; 368: 952-5

8. McTaggart RA, Jovin TG, Lansberg MG et al: Alberta stroke program early computed tomographic scoring performance in a series of patients undergoing computed tomography and MRI: reader agreement, modality agreement, and outcome prediction. Stroke, 2015; 46: 407-12

9. Ding J, Zhao Z, Wang $\mathrm{C}$ et al: Bioluminescence imaging of transplanted human endothelial colony-forming cells in an ischemic mouse model. Brain Res, 2016; 1642: $209-18$

10. Vidale S, Longoni M, Valvassori L, Agostoni E: Mechanical Thrombectomy in Strokes with Large-Vessel Occlusion Beyond 6 Hours: A Pooled Analysis of Randomized Trials. J Clin Neurol, 2018; 14: 407-12

11. Belayev L, Alonso OF, Busto R, Zhao W, Ginsberg MD: Middle cerebral artery occlusion in the rat by intraluminal suture. Neurological and pathological evaluation of an improved model. Stroke, 1996; 27: 1616-22, 1623

12. Longa EZ, Weinstein PR, Carlson S, Cummins R: Reversible middle cerebral artery occlusion without craniectomy in rats. Stroke, 1989; 20: 84-91

13. Belayev L, Busto R, Zhao W, Ginsberg MD: Quantitative evaluation of blood-brain barrier permeability following middle cerebral artery occlusion in rats. Brain Res, 1996; 739: 88-96

14. Zheng YQ, Liu JX, Li XZ, Xu L, Xu YG: RNA interference-mediated downregulation of Beclin1 attenuates cerebral ischemic injury in rats. Acta Pharmacol Sin, 2009; 30: 919-27

15. Nygren J, Wieloch T, Pesic J, Brundin P, Deierborg T: Enriched environment attenuates cell genesis in subventricular zone after focal ischemia in mice and decreases migration of newborn cells to the striatum. Stroke, 2006; 37: 2824-9

16. Ziemka-Nalecz M, Zalewska T: Endogenous neurogenesis induced by ischemic brain injury or neurodegenerative diseases in adults. Acta Neurobiol Exp (Wars), 2012; 72: 309-24 
17. Kaur C, Ling EA: Periventricular white matter damage in the hypoxic neonatal brain: role of microglial cells. Prog Neurobiol, 2009; 87: 264-80

18. Yang Z, Zhong L, Zhong S, Xian R, Yuan B: Hypoxia induces microglia autophagy and neural inflammation injury in focal cerebral ischemia model. Exp Mol Pathol, 2015; 98: 219-24

19. Qin L, Crews FT: NADPH oxidase and reactive oxygen species contribute to alcohol-induced microglial activation and neurodegeneration. $\mathrm{J}$ Neuroinflammation, 2012; 9: 5

20. Barone FC, Arvin B, White RF et al: Tumor necrosis factor-alpha. A mediator of focal ischemic brain injury. Stroke, 1997; 28: 1233-44

21. Wang X: Investigational anti-inflammatory agents for the treatment of ischaemic brain injury. Expert Opin Investig Drugs, 2005; 14: 393-409

22. Savitz SI, Erhardt JA, Anthony JV et al: The novel beta-blocker, carvedilol, provides neuroprotection in transient focal stroke. J Cereb Blood Flow Metab, 2000; 20: 1197-204

23. Finley JW, Gao S: A Perspective on Crocus sativus L. (Saffron) Constituent Crocin: A Potent Water-Soluble Antioxidant and Potential Therapy for Alzheimer's Disease. J Agric Food Chem, 2017; 65: 1005-20

24. Jiang Z, Gu M, Liu J, Li H, Peng J, Zhang Y: Anticancer activity of crocin against cervical carcinoma (HeLa cells): Bioassessment and toxicity evaluation of crocin in male albino rats. J Photochem Photobiol B, 2018; 180: 118-24

25. Khorasanchi Z, Shafiee M, Kermanshahi F et al: Crocus sativus a natural food coloring and flavoring has potent anti-tumor properties. Phytomedicine, 2018; 43: 21-7

26. Hire RR, Srivastava S, Davis MB, Kumar KA, Panda D: Antiproliferative Activity of Crocin Involves Targeting of Microtubules in Breast Cancer Cells. Sci Rep, 2017; 7: 44984

27. Algandaby MM: Crocin attenuates metabolic syndrome-induced osteoporosis in rats. J Food Biochem, 2019; 43: e12895

28. Huang A, Jia L: Crocin enhances hypothermia therapy in hypoxic 
ischemia-induced brain injury in mice. Acta Neurol Belg, 2019:

29. Clark WM, Lessov NS, Dixon MP, Eckenstein F: Monofilament intraluminal middle cerebral artery occlusion in the mouse. Neurol Res, 1997; 19: 641-8

30. Koizumi JI, Yoshida Y, Nakazawa T, Ooneda G. Experimental studies of ischemic brain edema:1. A new experimental model of cerebral embolism in rats in which recirculation can be introduced in the ischemic area. Jpnjstroke 1986, 8: 1-8.

31. Jung KH, Chu K, Ko SY et al: Early intravenous infusion of sodium nitrite protects brain against in vivo ischemia-reperfusion injury. Stroke, 2006; 37: 2744-50

32. Han J, Wan H, Li J, Ge L: [Study on 3'-methoxy puerarin to dynamic changes of amino acids in rat brain ischemia-reperfusion]. Zhongguo Zhong Yao Za Zhi, 2012; 37: 1023-7

33. Dai G, Chen Y, Gu F, Chen R: Effects of electroacupuncture on the contents of NO, ET and T-AOC in the brain tissues of the cerebral hemorrhage model rats. J Tradit Chin Med, 2005; 25: 62-5

34. Mathew S, Kumar KA, Anandan R, Viswanathan NP, Devadasan K: Changes in tissue defence system in white spot syndrome virus (WSSV) infected Penaeus monodon. Comp Biochem Physiol C Toxicol Pharmacol, 2007; 145: 315-20

35. Gulesserian T, Engidawork E, Fountoulakis M, Lubec G: Antioxidant proteins in fetal brain: superoxide dismutase-1 (SOD-1) protein is not overexpressed in fetal Down syndrome. J Neural Transm Suppl, 2001: 71-84

36. Li M, Zheng $\mathrm{Y}$, Liang $\mathrm{H}$ et al: Molecular cloning and characterization of cat, gpx 1 and $\mathrm{Cu} / \mathrm{Zn}$-sod genes in pengze crucian carp (Carassius auratus var. Pengze) and antioxidant enzyme modulation induced by hexavalent chromium in juveniles. Comp Biochem Physiol C Toxicol Pharmacol, 2013; 157: 310-21 


\section{Figure legends}

Figure 1. The structures of crocin I and II.

Figure 2. The changes in Glu (A), Tau (B), and GABA (C) concentrations in the frontal cortex in Model rats after crocin I and II intervention for ischemia and reperfusion $(\mathrm{ng} / \mathrm{mg}, \mathrm{n}=6)$. I-40: ischemia for $40 \mathrm{~min}$; I-70: ischemia for $70 \mathrm{~min}$; I-100: ischemia for $100 \mathrm{~min}$; I-100+R-40: ischemia for $100 \mathrm{~min}$, then reperfusion for $40 \mathrm{~min}$; I-100+R-70: ischemia for $100 \mathrm{~min}$, then reperfusion for $70 \mathrm{~min}$; I-100+R-200: ischemia for $100 \mathrm{~min}$, then reperfusion for $200 \mathrm{~min}$.

Figure 3. Antioxidant index results, including MDA (A), GSH-Px (B), T-AOC (C), and T-SOD (D), for the left-brain tissue (non-ischemic control side) and right-brain tissue (ischemic side). $\mathrm{n}=10 . *: P<0.05$ vs. the Sham group; \#: $P<0.05$ vs. the Model group.

Figure 4. Effects of crocin I and II on the mRNA levels of the Bcl2/Bax ratio (A), Casp3 (B), Nfkb1 (C), and p38 (D) in the prefrontal cortex of ischemia-reperfusion injury rats. $\mathrm{n}=3$. I-40: ischemia for $40 \mathrm{~min}$; I-100: ischemia for $100 \mathrm{~min}$; I-100+R-40: ischemia for $100 \mathrm{~min}$, then reperfusion for $40 \mathrm{~min}$; I-100+R-200: ischemia for 100 $\mathrm{min}$, then reperfusion for $200 \mathrm{~min}$. 


\section{Crocin I}

\section{Crocin II}
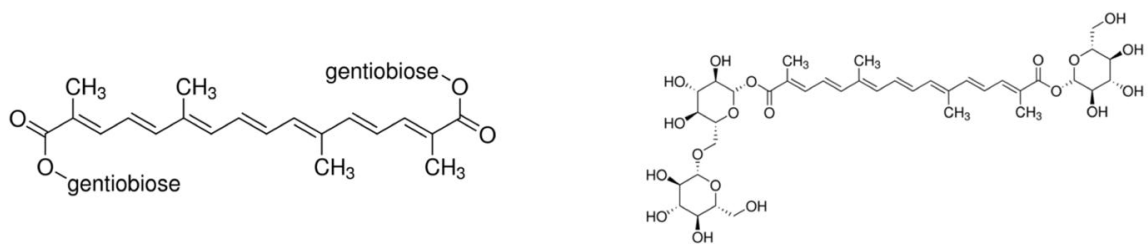


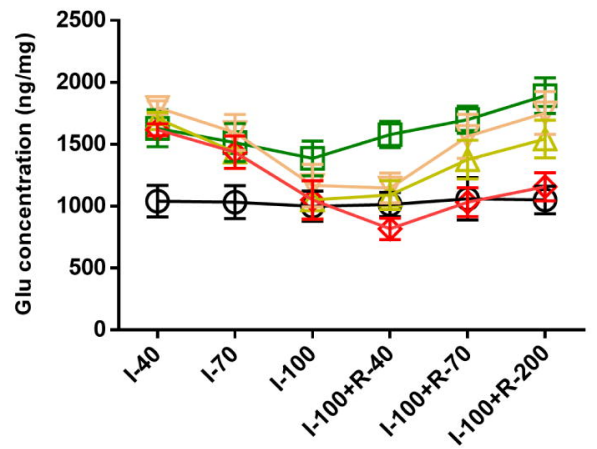

$\theta$ Sham

$\boxminus$ Model

F C1

$\triangle \mathrm{C} 2$

$\diamond \mathrm{C} 1+2$

Time points

B.

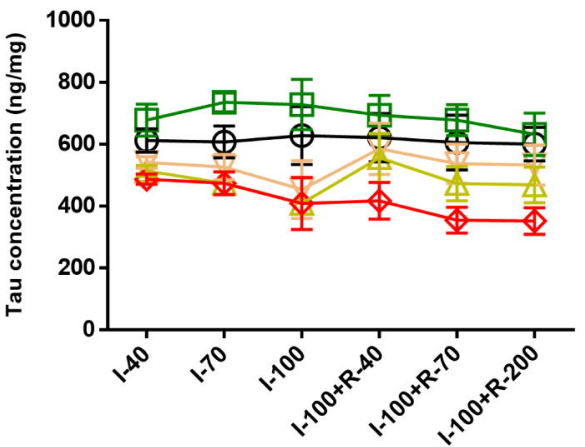

$\theta$ Sham

$\boxminus$ Model

F $\mathrm{C} 1$

$\triangle \mathrm{C} 2$

$\diamond \mathrm{C} 1+2$

Time points

c.

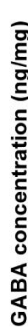

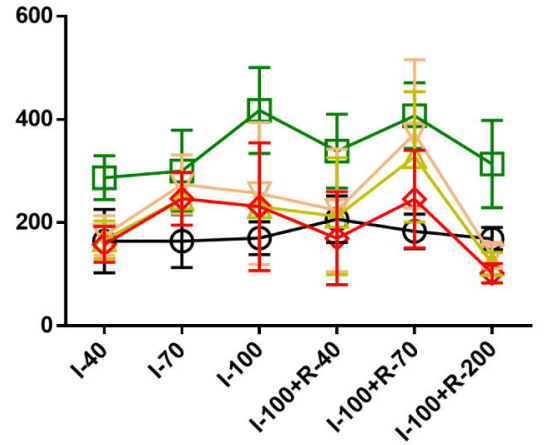

○ Sham

$\boxminus$ Model

F C1

$\triangle \mathrm{C} 2$

$\diamond \mathrm{C} 1+2$

Time points 
A.

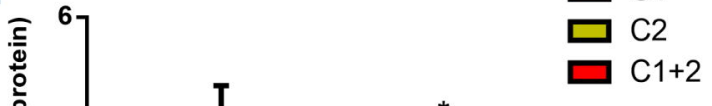

B.

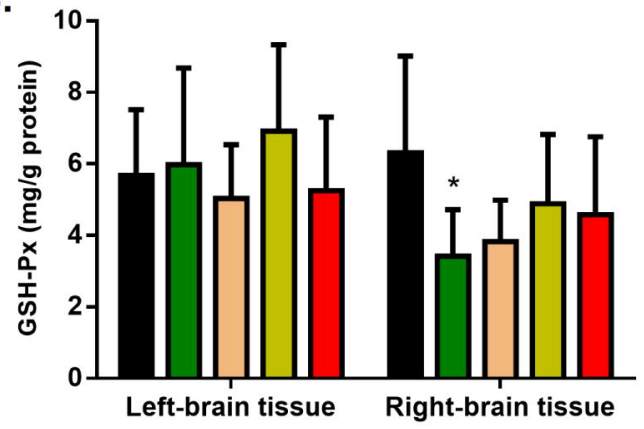

C.

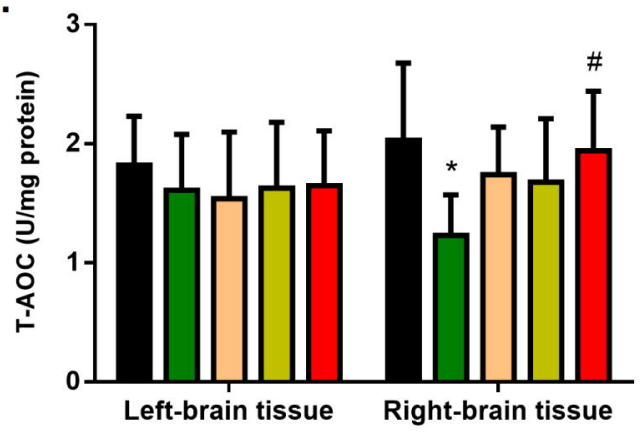

D.

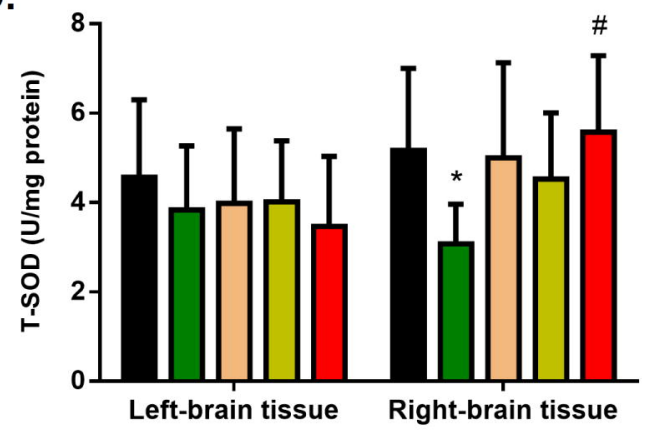


A.

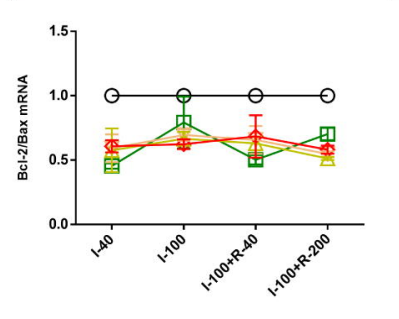

B.

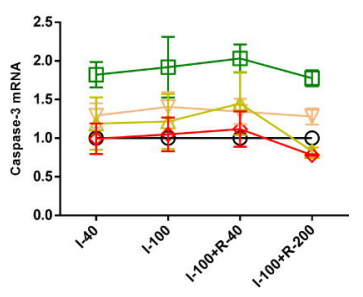

c.

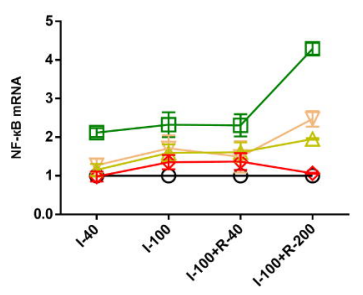

$\ominus$ Sham

$\forall$ Mod v.

D.

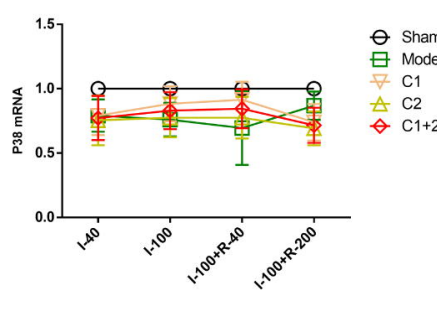

